\title{
Explanatory models and help-seeking behaviour of leprosy patients in Adamawa State, Nigeria
}

\author{
N. VAN DE WEG*, E. B. POST**, R. LUCASSEN*, \\ J. T. V. M. DE JONG† \& J. VAN DEN BROEK \\ *University of Maastricht, the Netherlands \\ **TBL Control Programme Adamawa State, Nigeria \\ $\dagger$ Transcultural Psychosocial Organisation, Amsterdam, \\ The Netherlands \\ Royal Tropical Institute, The Netherlands
}

Accepted for publication 10 September 1998

\section{Introduction}

The main strategy for leprosy control programmes consists of early case finding and treatment with multidrug therapy, ${ }^{1}$ which are still major challenges. ${ }^{2}$

The first decision of patients to go for care, and to whom to go, is influenced by many personal and social factors, such as gender, ethnic group, socio-economic status, costs, distance, availability of helpers and fear of stigmatization. ${ }^{3-6}$ Treatment choices are also influenced by the explanatory model the patients are using for their illness. ${ }^{7}$ Kleinman $^{3}$ defines the explanatory model as 'the notions about an episode of sickness and its treatment that are employed by all those engaged in the clinical process'. They are held by both patients and practitioners, and they 'offer explanations of sickness and treatment to guide choices among available therapies and therapists and to cast personal and social meaning on the experience of sickness'.

In 'traditional' societies, help seeking is often diverse, and the position of indigenous healers within the medical system is an important one. ${ }^{8}$ Although indigenous healers are competent in healing chronic and self limiting ailments as well as minor psychological disorders, this does not seem to be the case for severe, acute diseases. ${ }^{9}$

The medical system can be divided into three overlapping sectors. The popular sector comprises the lay, non-professional domain which includes self-medication, lay management and advice of relatives, friends, neighbours, village heads, religious leaders, other patients and chemists. The folk sector includes sacred and secular folk healers as well as barbers. The professional sector comprises the organized, legally sanctioned healing professions. ${ }^{3}$

In Adamawa State in Nigeria, the majority of the population choose modern medicine as

Correspondence to: E. B. Post, c/o: Netherlands Leprosy Relief Association, Wibautstraat 137-k, 1097 DN Amsterdam, The Netherlands 
their treatment of first choice, especially for a variety of chronic diseases. Traditional medicine followed closely, and was in some cases even preferred. ${ }^{10}$ In the case of leprosy, use of traditional treatments might be one of the factors causing delay.

In Adamawa State, approximately $20 \%$ of leprosy patients appear to have visible deformities at the time of diagnosis, pointing to late reporting of patients and thus to the need to strengthen case finding activities.

To improve case finding, it is important to understand what happens in the phase before patients contact modern health care facilities and how they label and understand their sickness. This study focuses on explanatory models and help seeking behaviour of leprosy patients.

The study was performed in 1994 in Adamawa State, in the north-eastern part of Nigeria. It is a rural area, known for its many ethnic groups, which all have their own language and customs. Hausa is a widely spoken second language. The registered prevalence rate of leprosy in 1993 was $4 \cdot 1 / 10,000$ and the case detection rate $1 \cdot 2 / 10,000$ population.

\section{Materials and methods}

A questionnaire was used that focussed on help-seeking behaviour and explanatory models. ${ }^{11}$ First signs and symptoms were asked for in an open question, leaving space for patients' explanatory model, after which an interpretation was done by the investigator following the lines of modern categories of dermatological symptoms, nerve function impairment, general symptoms and others. From the time these first signs and symptoms developed, each helpseeking step was explored: patients were asked to whom they went for treatment, who took the initiative to seek care and what kind of treatment they received. For each help-seeking step, the explanatory model the patient was using was elicited; this included questions about the labelling of their problem and perceived cause.

The study can be divided into two steps:

\section{INTERVIEWS WITH KEY INFORMANTS}

To gain a first impression about leprosy-associated beliefs at the population level, several key informants were interviewed: persons who occupy a position of respect and trust and have contact with many people within the community. Two folk healers (one male and one female), a village head, a schoolteacher, a Muslim leader and a Christian police of ficer were interviewed. The information thus obtained was used to adjust the questionnaire and to get a better understanding of the general socio-cultural context.

\section{INTERVIEWS WITH PATIENTS}

Patients $(n=60)$ were selected whose treatment duration at the time of the interview was 24 months or less and had never been treated before in a leprosy hospital or control programme. The majority of these patients $(n=49)$ was interviewed in 28 different outpatient clinics. The remaining 11 patients were interviewed at the leprosy hospital in Garkida.

Two Dutch medical students performed the interviews with the help of two interpreters. To increase inter-rater reliability, the first five interviews were done together. After 30 
interviews, the completed questionnaires were discussed in order to trace possible differences in interpretation of the questions, which were absent.

The interpreters were leprosy supervisors who were unknown to the patients. They were carefully instructed about the purpose of the study and the use of the questionnaire. Most patients were interviewed in Hausa. In 11 cases (18\%), a second interpreter had to be used. The duration of the interviews ranged from 45 to $120 \mathrm{~min}$.

Patients younger than 12 years were excluded.

\section{Results}

In Table 1, the characteristics of the patients in this study are summarized. Of the 60 leprosy patients, $15(25 \%)$ were classified as paucibacillary and $45(75 \%)$ as multibacillary.

\section{PERCEIVED CAUSES OF LEPROSY}

Table 2 summarizes the perceived causes of leprosy reported by leprosy patients answering an open-ended question. They answered the question after they already had received some form of health education.

An overwhelming majority of the patients $(58 \%)$ used traditional beliefs to explain the cause of leprosy. Food was perceived by 14 patients (23\%) as the main cause of leprosy. The foodstuffs most frequently mentioned were goat meat, groundnut and (mud-) fish, which are important ingredients of the day-to-day diet. They did not seem to be speaking about sorcery, neither was it taboo to eat these foodstuffs. Many patients had only stopped eating goat meat after they were told they had leprosy. Many respondents (27\%) perceived leprosy mainly as 'God's wish', without further explanation.

Sixty percent of the patients started treatment in the last 3 months before the interview, 15 patients $(25 \%)$ were diagnosed as leprosy patients less than 2 weeks before the interview.

The modern concept of contagion was less frequently mentioned (20\%). Ten leprosy

Table 1. Main characteristics of the interviewed patients

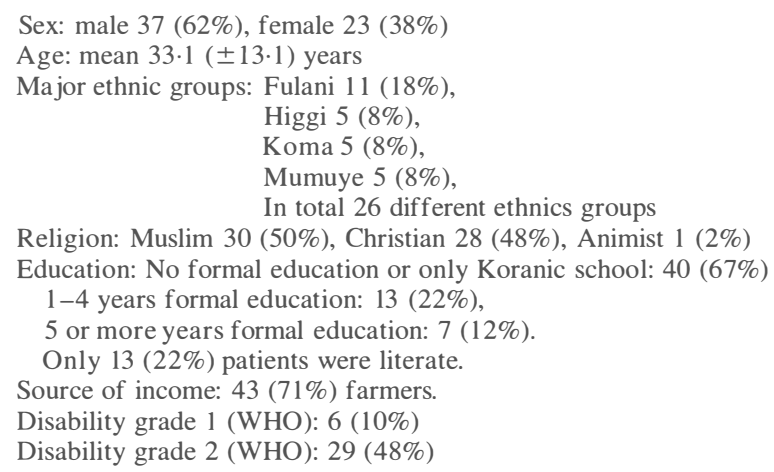


Table 2. Perceived causes of leprosy by leprosy patients ( $n=60$, first answer counted). Fisher's exact (male/female) $=0.422$

\begin{tabular}{|c|c|c|c|}
\hline Cause & $\begin{array}{c}\text { Male }(n=37) \\
(\%)\end{array}$ & $\begin{array}{c}\text { Female }(n=23) \\
(\%)\end{array}$ & $\begin{array}{c}\text { Totals }(n=60) \\
(\%)\end{array}$ \\
\hline Traditional beliefs (food, god, witchcraft, taboo, spirits) & $19(51)$ & $16(70)$ & $35(58)$ \\
\hline Modern concepts (contagion) & $9(24)$ & $3(13)$ & $12(20)$ \\
\hline Other (heredity, hygiene, physical) & $5(14)$ & $1(4)$ & $6(10)$ \\
\hline Don't know & $4(11)$ & $3(13)$ & $7(12)$ \\
\hline Total & $37(100)$ & $23(100)$ & $60(100)$ \\
\hline
\end{tabular}

patients considered germs or bacteria to be the cause of leprosy, other patients mentioned 'contact with leprosy patients' (eating together, bathing in the same water, etc.).

Most respondents (65\%) thought everybody could get leprosy, $15 \%$ thought only some persons could get the disease, for reasons closely connected to their perceived cause, while $8 \%$ were not sure. Twelve percent of the respondents thought that each person had the disease in the body, but that in most cases the disease didn't 'come out'.

No significant difference was found between male and female patients concerning their concept of leprosy (Fisher's exact $=0.422$ ).

DELAY AND HELP-SEEKING

The duration of delay between noticing the first signs and symptoms and the first contact with the leprosy services ranged from 0 to 10 years, with a median total delay of 36 months. In the majority of the cases $(68 \%)$, more than a year passed before the patient started effective treatment. Once a lay diagnosis of leprosy was made, $27 \%$ of the patients found their way to the leprosy services within 3 months. The median delay after the lay diagnosis was 12 months.

Multivariate analysis using Cox regression analysis showed no significant correlations between delay on the one hand and sex, age, religion, level of education or leprosy classification on the other. There were correlations, however, with visible deformity at the time of interview ( $p=0.008$ with a $95 \%$ confidence interval of $0.413-$ 0.876 ) and illiteracy ( $p=0.019$ with a 95\% confidence interval of $0.064-0.785$ ). No difference in delay was found between patients with either grade 0 and grade 1 disability (WHO). The delay in diagnosis was, however, consistently larger among patients with visible deformities at the time of interview, as evidenced by a Kaplan Meier analysis (Figure 1).

The flow diagram (Figure 2) shows to whom people went for treatment or advice before they started modern treatment for leprosy. Surprisingly, 19 (32\%) respondents went straight to the leprosy services without another help-seeking step in between: women (43\%) more so than men (24\%). The folk sector was the most frequently [22 times (37\%)] consulted as a first step, followed by the popular sector [14 times (23\%)]. The professional sector was consulted as a first step only 5 times $(8 \%)$.

Although folk healers denied treating leprosy and said that they referred such patients, in fact none of their consultations resulted in referral. Their treatment ranged from modern medicines to herbal treatments, dietary advice, hygienic measures, to 'writings', where 


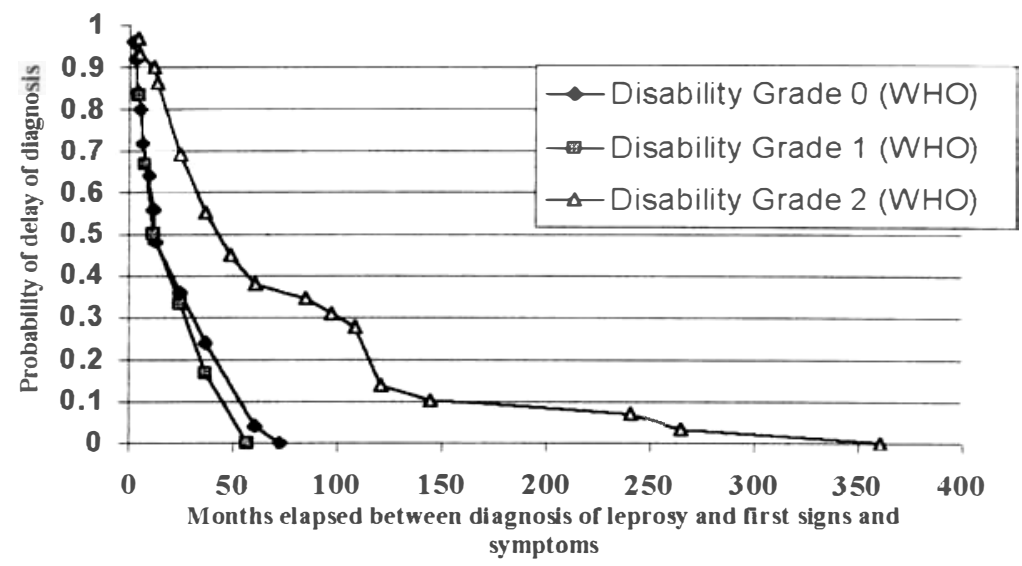

Figure 1. Kaplan Meier analysis of the delay of diagnosis of leprosy since first signs and symptoms.

Koranic verses were written on a wooden slate and washed off with water which was given to the patient to drink.

The total number of help-seeking steps ranged from one to six steps; most patients had a total number of two (27\%) or three (32\%) help-seeking steps; only $11 \%$ had four or more help-seeking steps before reaching the leprosy services.

In general, patients tended to consult different practitioners one after the other; rarely did they use two treatments at the same time. When they evaluated their treatment as not effective, they usually 'tried their luck' somewhere else. There was no clear pattern in the sequence of practitioners consulted.

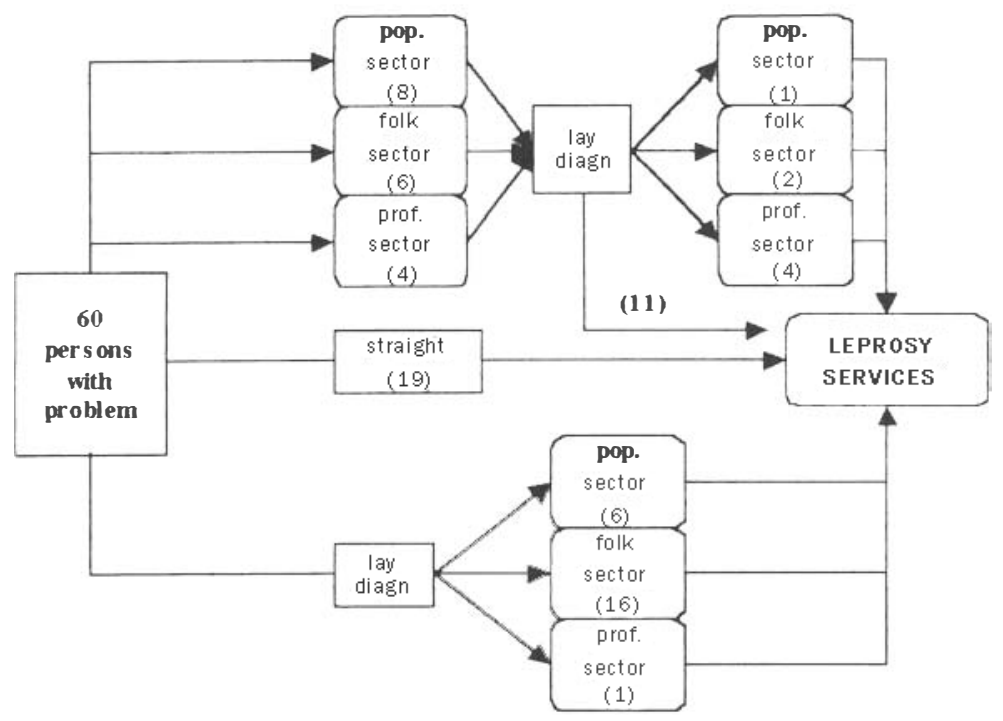

Figure 2. Where leprosy patients go with their (leprosy) problem before starting modern treatment. 


\section{Discussion}

This study has shown that a majority of the patients had a total delay of more than a year from the first signs and symptoms to consultation of the leprosy services. A longer delay was correlated with visible deformities and illiteracy.

The large proportion of patients labelling their problem as leprosy before their first contact with leprosy services and the high percentage of diagnoses being made by others than the health workers suggests that many people in the community are aware of the early signs and symptoms of leprosy. In contrast with other studies performed in India ${ }^{12}$ and Pakistan, ${ }^{13}$ patients rarely denied their diagnosis of leprosy. A majority of the patients thought their condition would get worse if they did not go for treatment. This shows that unawareness of possible deterioration is not a likely explanation for delay. Most thought leprosy could be cured with modern treatment, but could not contact the leprosy services in an early stage, resulting in a high proportion of disabilities ( $48 \%$ visible disabilities in this study).

Consultation of other practitioners seemed to be the main reason for delay. The finding that many patients consulted other practitioners before reporting to leprosy services is in line with other studies. ${ }^{12-14}$ The professional sector seemed to be under-consulted, but we lack information on help-seeking behaviour for other ailments. It is our impression, however, that peripheral health units were generally under-utilized, to which factors like costs and poor availability of essential drugs might have contributed.

In this study, no significant difference in delay could be found between men and women. The lower number of registered female leprosy patients can thus not be easily explained by possible women's restricted mobility, as was found in Nepal. ${ }^{4}$ Other factors have to be looked into. Ethnic differences in help-seeking behaviour have been found in Nepal, ${ }^{4}$ but could not be analysed in this study, due to the large number of ethnic groups in Adamawa State. ${ }^{15,16}$ General considerations as expressed in the 'etic-emic' debate ${ }^{17}$ also point at the significance of the cultural context in relation to people's view on sickness and consequently sickness behaviour. In this study, however, no significant difference was found between male and female patients concerning their concept of leprosy.

The fact that only few patients report personal problems or fear of discrimination suggests that leprosy patients in Adamawa State are not severely stigmatized, which has been observed by others as well. ${ }^{18,19}$ This contrasts with the study of Mull et al. ${ }^{13}$ in Pakistan, where $54 \%$ spoke of severe personal problems.

Comparison with other studies shows that perceived causes of leprosy differ from culture to culture.

Food was a frequently mentioned cause in Pakistan, ${ }^{13}$ Bombay ${ }^{12}$ and northern Nigeria, but not in Thailand. ${ }^{14}$ Whereas in Pakistan, patients explained this as an imbalance between 'hot' and 'cold', this did not seem to be the case in northern Nigeria, nor did sorcery or a taboo seem to be the underlying reason. ${ }^{18}$

The high number of respondents attributing the disease to God was specific for northern Nigeria. Lewis Wall ${ }^{18}$ described leprosy as one of the 'Ciwon Allah' (diseases of God), 'regarded as a not quite comprehensible manifestation of the power of God and accepted as such'. This is in line with the (official) Muslim view, where all events, including disease, are caused by the will of God irrespective of causes, but are not considered as punishment by God for sins or wrong-doings. ${ }^{20}$ Whereas in Nepal one study suggested that fear of stigmatization led patients to travel farther for treatment in order to disguise their diagnosis, ${ }^{4}$ this did not seem to be the case in this study. Most patients sought treatment at their local clinic. 
Contagion was a frequently mentioned cause in northern Thailand, ${ }^{14}$ Bombay ${ }^{12}$ and northern Nigeria.

\section{Conclusion}

Based on the findings in this study, several measures can be taken to improve early case finding. First of all, it seems to be important to involve folk healers in case finding activities. They are the ones most frequently consulted as an alternative to the leprosy services.

The lay referral system (the general public and chemists) could be further exploited by improving health education, not merely about early signs and symptoms of leprosy but also about where to go when leprosy is suspected. For example, the use of radio programmes would not only reach a wide audience but would also address illiteracy as an obstacle in finding the leprosy services.

\section{Summary}

In northern Nigeria 60 leprosy patients, 49 outpatients and 11 in-patients, were interviewed about their help-seeking behaviour and explanatory models before their first contact with the leprosy services. Most patients showed a delay of more than 1 year. After leprosy was provisionally diagnosed by lay persons, $27 \%$ of patients found their way to the leprosy services within 3 months. Chemists (popular sector) and the professional sector frequently missed the diagnosis. If early case finding is to be improved, it is important to involve them in case finding activities and to train them in adequate diagnostic skills.

No significant correlations were found between total delay and sex, age, religion or leprosy classification, except with visible deformity at the time of the interview and illiteracy.

Consultation of folk healers was the major reason for delay. Most patients consulted folk healers, who, although they claimed to have a positive attitude towards modern medicine in the case of leprosy, never referred patients to the leprosy services.

While many patients held a variety of causes responsible for leprosy, most patients explained the disease in traditional terms $(58 \%)$, while only a minority used modern concepts (20\%). This emphasizes the need for continuous attention for health education of diagnosed patients and their families. No significant difference was found between male and female patients concerning their concept of leprosy.

Denial of the leprosy diagnosis was rare.

\section{Acknowledgements}

This study was supported by a grant from the Stichting Wetenschappelijk Onderwijs Limburg. The authors are particularly grateful to Mr Thomas Abalan and Mr Satburi Jared for their excellent interpretations of the interviews. Also, we wish to express our warmest thanks to the many leprosy patients who shared their time and stories to help us learn from their experiences. 


\section{References}

1 WHO. A guide to leprosy control. WHO, Geneva, 1988.

${ }^{2}$ Sasakawa Memorial Health Foundation. Leprosy profiles with special attention to MDT implementation. SMHF/MDT Series 2, SMHF, Tokyo, 1991.

3 Kleinman A. Patients and healers in the context of culture. University of California Press, Berkeley, 1980.

${ }^{4}$ Pearson M. What does distance matter? Leprosy control in West Nepal. Soc Sci Med, 1988; 26: 25-36.

5 Varkevisser CM. Psychological factors relevant to leprosy control. Proceedings of the scientific conference on the epidemiology of leprosy, Arusha, Tanzania, 1978: 109-118.

6 Zola IK. Pathways to the doctor-from person to patient. Soc Sci Med, 1973; 7: 677-689.

7 Helman CG. Culture, health and illness. Butterworth-Heinemann, Oxford, 1990.

${ }^{8}$ Leslie C. Medical pluralism in world perspective. Soc Sci Med, 1980; 14B: 191-195.

9 Kleinman A, Sung L. Why do indigenous healers successfully heal? Soc Sci Med, 1979; 13, 7-26.

10 Padonu MKO, Olowokure BO, Birmah U et al. Traditional medicine in health care delivery system in Gongola State: the case of Song Local Government Area. Annals of Borno, 1988; 5: 160-169.

11 De Jong, JTVM. Questionnaire for help-seeking behaviour and explanatory models. IPSER, Maastricht, 1992.

12 Weiss MG, Doongaji DR, Siddhartha S et al. The Explanatory Model interview catalogue (EMIC). Contribution to cross-cultural research methods from a study of leprosy and mental health. Br J Psych, 1992; 160: 819-830.

13 Mull JD, Shear Wood C, Gans LP, Mull DS. Culture and 'compliance' among leprosy patients in Pakistan. Soc Sci Med, 1989; 29: 799-811.

${ }^{14}$ Neylan TC, Nelson KE, Schauf V, Scollard DM. Illness beliefs of leprosy patients: use of medical anthropology in clinical practice. Int J Lepr, 1988; 56: 231-237.

15 Capro Research Office. The cross and the gods: a look at Adamawa and Taraba States, Nigeria. Baraka Press and Publishers, Kaduna, Nigeria, 1992.

16 Nissen M. An African Church is born. The story of the Adamawa and Central Sardauna Provinces in Nigeria. Reprinted by Betel \& Partners, Nigeria, 1993.

17 Richters JM, The medical anthropologist as a narrator and a translator: on a journey with Hermes in the land of idols. Society for Medical Anthropological Research \& Theory. Eburon, Delft, The Netherlands, 1991: 234-240.

${ }^{18}$ Lewis Wall L. Hausamedicine. Illness and well-being in a West African culture. Duke University Press, Durham, 1985.

19 Shiloh A. A case study of disease and culture in action: leprosy among the Hausa of northern Nigeria. Hum Org, 1965; 24: 140-147.

20 Mohamed HAA. Leprosy—the Moslem attitude. Lepr Rev, 1985; 56: 17-21. 